\title{
Determination of Stress Concentration Factors in Offshore Wind Welded Structures through a Hybrid Experimental and Numerical Approach
}

\author{
Athanasios Kolios ${ }^{1}$, Lin Wang ${ }^{2}$, Ali Mehmanparast ${ }^{3}$, Feargal Brennan ${ }^{1}$ \\ ${ }^{1}$ Department of Naval Architecture, Ocean \& Marine Engineering, University of Strathclyde, \\ Glasgow, United Kingdom \\ ${ }^{2}$ School of Mechanical, Aerospace and Automotive Engineering, Coventry University, Coventry, \\ United Kingdom \\ ${ }^{3}$ Offshore Energy Engineering Centre, Cranfield University, Cranfield, Bedfordshire, United \\ Kingdom
}

Abstract: Offshore wind turbine (OWT) monopile support structures generally consist of steel cans connected together through circumferential welding joints. One critical factor to evaluate the localised increase in stresses is the stress concentration factor (SCF) which depends on the welding quality. The complex welding profiles in OWT monopiles makes the accurate calculation of SCF quite challenging. In this work, an innovative approach for the calculation of SCFs in offshore welded structures is proposed based on combined 3D (three-dimensional) laser scanning technology (LST) and 3D finite element analysis (FEA). The precise geometry of the welded specimens is captured using $3 \mathrm{D}$ LST, and then imported into a finite element software to perform 3D FEA modelling to accurately calculate SCFs. A 2D (two-dimensional) FEA model of a typical offshore welded structure with ideal geometry is also developed in this work. In addition to numerically calculate SCFs, the 2D FEA model is further combined with non-linear RSM (response surface method) to derive analytical equations, expressing SCFs of offshore welded structures in terms of key welding parameters. Both LST-FEA3D and RSMFEA2D models are applied to calculate SCFs in large-scale S-N fatigue welded specimens. The results indicate that the LST-FEA3D approach is capable of capturing the variation of SCFs along the width of the welded specimens and identifying the critical points where fatigue crack is most likely to initiate; and the RSM-FEA2D is valuable and efficient in deriving analytical parametric equations for SCFs.

Keywords: Stress concentration factor; Offshore wind monopiles; Steel structures; Parametric equations; FEA modelling 


\section{Introduction}

A realistic scenario from Wind Europe foresees $320 \mathrm{GW}$ of wind energy capacity to be installed in the EU by 2030, with significant contribution of $66 \mathrm{GW}$ from offshore wind farms (EWEA, 2015). Offshore installations benefit from higher wind speeds, unrestricted space, and lower social impact in the marine environment (Lozano-Minguez et al., 2011). Key barriers that need to be overcome towards further deployment of offshore wind farms are the high construction cost, especially foundation and electrical connection, and limitations in operation and maintenance which constitute a considerable part of life cycle costs (loannou et al., 2018).

The substantial wind potential in Europe has led over the last years to a considerable increase in operational wind farms, with the UK having the highest installed capacity with farms in the North Sea, Irish Sea and Baltic Sea (Kallehave et al., 2015), with plans for significant expansion in the next decade. An OWT generally comprises of a wind turbine installed on top of a structure which is resting on a foundation that is embedded in the soil transferring loads. For the efficient service life operation of these assets it is important to accurately estimate the acting loads and their resulting effects in order to evaluate their integrity and hence their residual service life. There are various types of support structures that can accommodate OWTs, while selection of the most appropriate configuration depends on a number of criteria including the water depth, the estimated environmental loads, the cost of production and installation, complexity of the design etc (Kolios et al., 2016, 2010). The monopile support structure configuration is currently adopted in most existing projects in Europe due to its simple but robust design (Gentils et al., 2017), ease of fabrication and installation. Monopiles were deemed to be economically and technically feasible for water depths of less than $30 \mathrm{~m}$ (Seidel, 2010); however, the limits of their applicability has shifted and monopiles have been recently deployed in water depths of around $40 \mathrm{~m}$ (Peeringa, 2016).

OWT monopiles are generally made of hot-rolled structural steel plates subjected to coldrolling followed by welding in the longitudinal direction to form "cans". The individual cans are subsequently welded circumferentially to fabricate a full-length monopile (A. Mehmanparast, O. Adedipe, F. Brennan, 2016). Several types of welded joints exist, e.g. single-V butt joint, double- $\mathrm{V}$ butt joint, corner joint, lap joint, etc. The double- $\mathrm{V}$ butt joint, in which $\mathrm{V}$-shape welds are on both sides of the work piece, is generally used in OWT monopiles. The state of stress of those welded joints is complex and affected by several factors, such as residual stresses and welding profiles. Welding quality can significantly affect the structural performance of OWT monopiles, as high stresses local to the welded joints could result in crack initiation and failure in monopiles. One critical factor to evaluate the stress and quality of welded joints is 
the SCF (stress concentration factor), which is defined as the ratio of the local stress at the welded region to the nominal stress. It should be noted that different types of welded joints have different welding profiles and therefore the associated SCFs are also different and vary across the weld due to the welding process inherent variability. When calculating SCFs for different welded joints, it is important to consider the corresponding welding profiles.

Approaches used to calculate SCFs can be categorised into three groups, i.e. experimental evaluations, empirical equations and FEA (Finite Element Analysis). In the experimental evaluations, the local strain/stresses at the weld toe are measured with strain gauges. The nominal stress can be obtained from experimental measurements or analytical calculations. Experimental measurements are difficult and not always possible to put strain gauges close enough to the weld toe to obtain meaningful results. Experimental methods also tend to be expensive and time-consuming in cases of complicated weld profiles (Pirali, 2006). Another way to obtain SCFs is to use the empirical equations, which are generally given by design standards, such as API-RP2A-WSD (American Petroleum Institute, 2014) and DNVGL-ST0126 (DNV GL AS, 2016). The empirical equations are efficient in cases of simple geometries; however, they are valid only for a limited range of non-dimensional geometric parameters and incapable of identifying the location of the critical points where fatigue cracking is most likely to initiate. An alternative way to obtain SCFs is to use FEA modelling, which is a powerful method to identify the locations of critical points and is ideally suited for the calculation of SCFs in complex geometries. Due to its accuracy and high fidelity, FEA has been increasingly used for the calculation of SCFs in offshore welded structures (Woghiren and Brennan 2009, Hellier et al 2014, Ahmadi et al., 2011a, 2011b; Lee, 1999). Considering its accuracy, the FEA approach is chosen in this study to calculate SCFs in offshore welded structures.

Reviewing recent work related to the calculation of SCFs for offshore applications, it is found that most research has taken place for tubular components and joints, and particularly for oil \& gas applications. More specifically, (Zhang et al., 2018) developed a novel framework for deriving the unified SCFs by reducing joint modelling from multi-planar out-of-plane overlapping to equivalent uniplanar non-overlapping. (Méndez et al., 2017) have performed FEA simulations and fatigue behaviour evaluation through employing 3D T-welded connections with intact and grinding depth conditions for A36 steel plates. (Wu and Chen, 2017) compared fatigue lives predicted by the fracture mechanics and S-N approaches for a floating spar structure, investigating the impact of the variation of initial crack depth, critical crack depth and SCFs on the ratio of the fatigue life predicted by two approaches. (Ahmadi and Zavvar, 2016) investigated the effect of multi-planarity on the SCFs in offshore tubular 
KT-joints subjected to in-plane and out-of-plane bending loads extracting results from the stress analysis of FEA models, verified against available experimental data, and performing a geometrically parametric investigation followed by a set of nonlinear regression analyses to derive a SCF parametric equation for the fatigue analysis and design. Further, (Ahmadi, 2016) derived a probability distribution model for SCFs in internally ring-stiffened tubular KT-joints of offshore structures subjected to out-of-plane bending loads. In (Ahmadi et al., 2016), based on results from the FEA analysis of 108 models, validated using test data obtained from the experimental investigation, probability distribution models for SCFs in internally ring-stiffened tubular KT-joints under four types of in-plane bending (IPB) loads were proposed. (Christiansen and Tang, 2016) have employed neural networks for tubular joint optimization in offshore jacket structures where influence factors (INF) and SCFs were applied to simplified models of relevant tubular joints in global models in order to achieve a realistic force flow in the structure. (Maheswaran and Siriwardane, 2016) have presented a comparative study of the fatigue life of tubular joints in offshore jackets according to the SCFs in DNV/GL-RP-0005 and FEA modelling. (Yang et al., 2015) studied SCFs of negative large eccentricity tubular Njoints under axial compressive loading in vertical brace through a combination of experiments and FEA simulations. (Ahmadi and Lotfollahi-Yaghin, 2015) calculated SCFs due to in-plane bending (IPB) loads in ring-stiffened tubular KT-joints of offshore structures through a parametric study extracting data from the FEA of 118 models, which were verified using test results obtained from an experimental investigation, to study the effect of geometrical parameters on the weld-toe SCFs of central and outer braces in internally ring-stiffened tubular KT-joints subjected to four different types of IPB loads. (Schmidt et al., 2015) performed fatigue reliability analysis for brace-column connection details in a semisubmersible hull applying SCFs for the selected critical hot spots, applied to the nominal component stresses due to axial forces and biaxial bending and using the hot-spot stress response spectra with various spectral methods to estimate fatigue damage using Miner's rule. (Lozano-Minguez et al., 2014) presented a comparison between the fatigue life predictions obtained by the SCFs of 3D solid FEA models considering the weldment and the existing SCF parametric equations for tubular T-joints. (Ogeman et al., 2014) presented a review of different direct calculation procedures to obtain the SCF based on fatigue assessment guidelines studying the uncertainty in SCF computation for ship fatigue design. (Li et al., 2014) presented a numerical study on girth weld of marine steel tubular piles deriving theoretically and validating the SCF at pipe splice under either axial tension or in-plane bending moment. (Cao et al., 2013) employed FEM methods to study the stress concentration factors of $\mathrm{K}$-joints with welding residual stress. (Ahmadi et al., 2013) presented results of experimental and numerical investigations of the chord-side SCF distribution of central brace in internally ring-stiffened 
tubular KT-joints. (Ahmadi and Lotfollahi-Yaghin, 2013) studied the effect of SCFs on the reliability of tubular joints through constructing a database using the results of FEA carried out on the right-angle two-planar tubular DKT-joints. Finally, (Chung et al., 2013) presented a methodology followed in the offshore industry for the prediction of the pile fatigue induced by pile hammering during installation. From this review, it can be concluded that although sufficient work is present for tubular joints and FEA is often employed for parametric studies, less focus has been given to simpler but thicker geometries which are particularly relevant to offshore wind energy applications.

The FEA models used for calculating SCFs can be further categorised into two groups, i.e. 1) 2D (two-dimensional) FEA, in which the stress changes in the thickness direction are neglected; and 2) 3D (three-dimensional) FEA, which considers the 3D geometry of specimens and capable of taking account of stress changes in the thickness direction. When comparing the 3D FEA, the 2D FEA is more computationally efficient. In cases that welding profiles remain unchanged along the thickness direction, the 2D FEA is capable of providing same results as the 3D FEA. However, for a practical welded specimen, the deviation of the welding profiles in the thickness direction is inevitable due to inherent complexity of the welding process. In order to capture the deviation of welding profiles in the thickness direction, it is necessary to use 3D FEA.

The accuracy of 3D FEA highly depends on the accuracy of the geometry used in the 3D FEA modelling, and the inaccuracies in the geometry can lead to a significant discrepancy between the FEA results and experimental data. Offshore welded structures generally have complex weld profiles, making the acquisition of the precise geometry of specimens used in the fatigue test quite challenging. In order to obtain precise geometries containing the full characterisation of weld toe profiles, it is proposed to use 3D LST (laser scanning technology) (E. Angelopoulou and J. R. Wright Jr, 1999; J. Beraldin, F. Blais, 2010), which is capable of acquiring a dense $3 \mathrm{D}$ coordinate information effectively and precisely over the complex surfaces of the weld toe.

To the best of authors' knowledge, the combination of LST and 3D FEA for SCF calculations in offshore welded structures cannot been found in the literature. In this work, a LST-FEA3D model, which combines 3D LST and 3D FEA, is developed for the calculation of SCFs in offshore welded structures. In the LST-FEA3D model, the precise geometry of the welded specimens is obtained using 3D LST, and then imported into a finite element software package to perform FEA modelling to calculate SCFs. The proposed approach is applied to calculate SCFs in large-scale welded specimens tested under cyclic loading to failure. The variation of SCFs along the width of the welded specimens is presented and compared to identify the 
range of SCFs in actual test specimens. Additionally, a RSM-FEA2D model, which combines 2D FEA with non-linear RSM (response surface method), is also developed in this work. In the RSM-FEA2D model, the 2D parametric FEA model of welded specimen developed in this work is used to perform a large number of 2D FEA simulations by varying the welding parameters within given limits. Multivariate regression is then used to post-process the $2 \mathrm{D}$ FEA results to establish the response surface, deriving analytical expression of SCFs in offshore welded structures. The LST-FEA3D model is used for capturing actual welding profile of fabricated specimens and examining detailed SCF distributions along the weld region. The RSM-FEA2D model is used for deriving analytical equations to calculate SCFs. Both LSTFEA3D and RSM-FEA2D models have been developed in this work for a comprehensive investigation on SCF calculations of offshore welded monopile structures. The RSM-FEA2D model is validated through case studies and then compared against the LST-FEA3D model.

This paper is structured as follows. Sections 2 and 3 present the development of the LSTFEA3D and RSM-FEA2D models, respectively. Section 4 presents the validation, results and discussion, followed by conclusions in Section 5 .

\section{LST-FEA3D (Combined 3D LST and 3D FEA)}

A LST-FEA3D model for the calculation of SCFs in offshore welded structures is developed by combining 3D LST and 3D FEA, of which details are presented below.

\subsection{D LST}

In order to capture the specimen geometry in full detail, a 3D scan-to-CAD system (as shown in Fig. 1) is used. The 3D laser scanner is equipped with a high-resolution camera. The scanning resolution used in this analysis is $0.2 \mathrm{~mm}$ (following a convergence study), which enables the analysis to be completed with sufficiently high accuracy and at a reasonable requirement of data storage. A portable scan model EXAscan ${ }^{\mathrm{TM}}$ and $3 \mathrm{D}$ data acquisition software Vxelement ${ }^{T M}$ are used to obtain the $3 D$ scan data. A 3D scan data processing platform, Geomagic Studio, is then used to create CAD models from the 3D scan data.

The 3D laser scanning procedure comprises three main steps, i.e. pre-processing, geometry capturing and post-processing, which are detailed below:

i. Pre-processing: In this step, the specimen is cleaned prior to 3D laser scanning to remove paint/dust/grease from the surface. 
ii. Geometry capturing: Having cleaned up the specimen, the scanning targets are positioned on the outer surface of the specimen, and then 3D scanning is performed by holding the trigger within the allowable range specified and moving it across the geometry.

iii. Post-processing: Once 3D scanning is completed, the captured data are postprocessed by the software. The post-processing starts by reducing noise from captured data using relevant routines. The geometry alignment is then fixed with respect to the $X, Y$ and $Z$ coordinate axes defined in the software. Once the specimen geometry is generated by the software, the model is saved in an STL format and then converted to IGS format, which is compatible with CAD and FEA software packages. An example of the scanned geometry model is illustrated in Fig. 2.

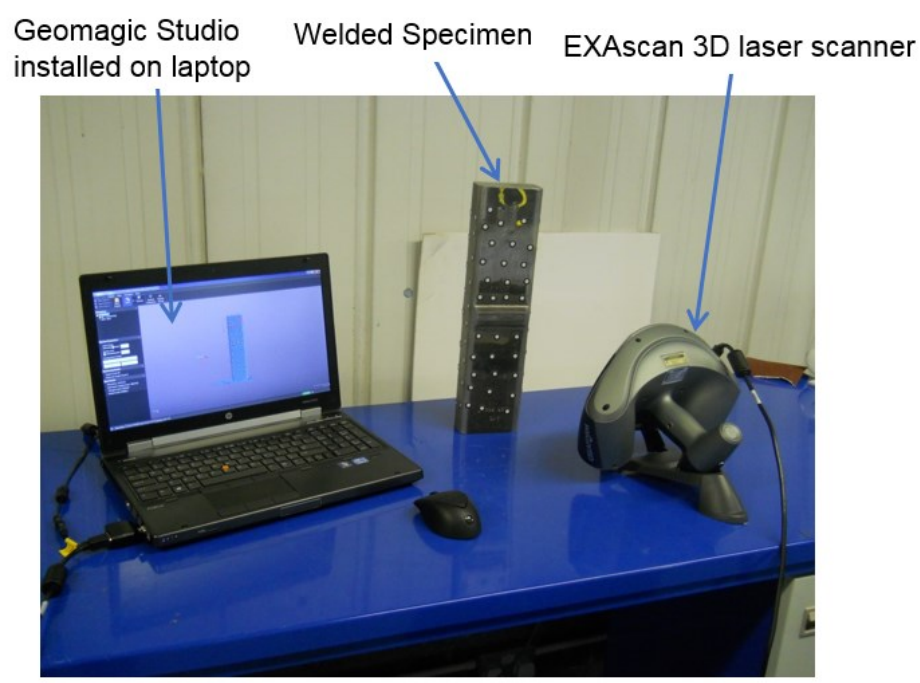

Figure 1. Setup of 3D scan-to-CAD system

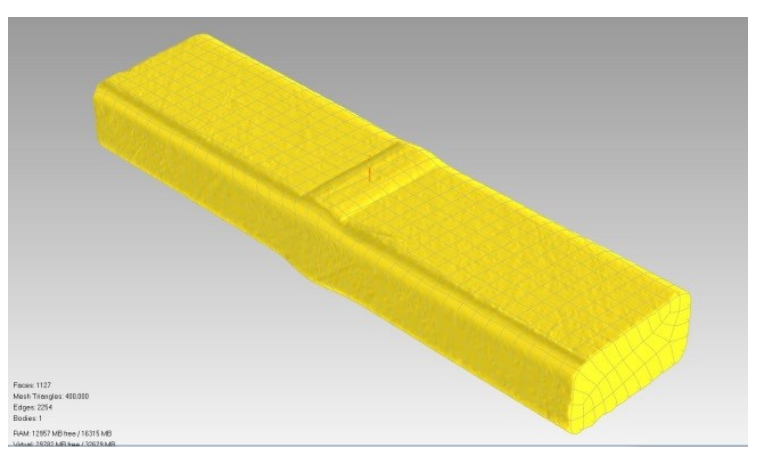

Figure 2. Scanned geometry model 
217 A 3D FEA model for scanned specimens is developed using Abaqus, which is a widely used

218 FE commercial software package. The geometry, material properties, mesh and boundary conditions used in the 3D FEA modelling are presented below.

\subsubsection{Geometry}

The scanned geometry is imported into Abaqus. At this stage irregular facets can be observed at both ends of the imported geometry, making it difficult to apply appropriate boundary conditions at both ends of the geometry. Cutting planes are used to trim the imported geometry, removing the irregular facets at both ends of the imported geometry and obtaining a flat plane at both ends of the geometry. The geometry after trimming is presented in Fig. 3a. At this stage a local coordinate system is defined, of which $\mathrm{x}$ and $\mathrm{y}$ axes are on the bottom plane of the specimen and the $z$ axis is perpendicular to the bottom plane in order to reconcile between different coordinate systems across the different tools employed. The local coordinate system is depicted in Fig. 3b.

230

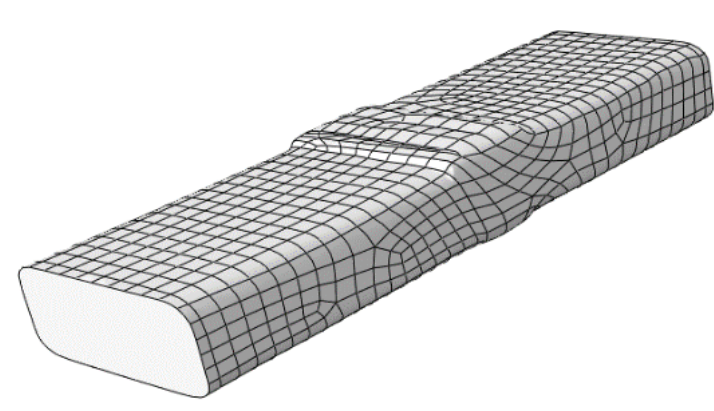

(a)

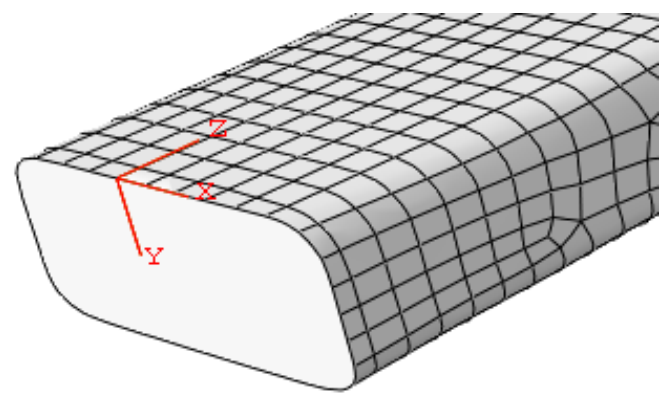

(b)

Figure 3. Geometry model: (a) geometry after trimming, (b) Local coordinate system

\subsubsection{Material properties}

The specimen is made of steel S355 which is a typical material used for offshore wind turbine monopiles (Igwemezie et al., 2018) with the Young's modulus of around $210 \mathrm{GPa}$ and Poisson's ratio of 0.3 (Mehmanparast et al., 2018). It is worth noting that the calculated SCF values only depend on the geometry of the welded joint and are independent of the materials properties. 
240 The scanned geometry captures the details of the welding profile; however, it also makes the 241 mesh generation quite challenging. The inherent irregular profiles of welded toes make it 242 impossible to generate structured meshes as they cannot capture the geometrical details of 243 interest. Therefore, unstructured mesh is used for the complicated welded geometries 244 examined in this work. In order to determine the proper mesh size at the welding region, mesh 245 sensitivity studies were performed. In this case, Specimen A, which was experimentally tested 246 in this work with a load range of $875 \mathrm{kN}$, is chosen as an example. Four mesh sizes at the 247 welding regions are studied, i.e. $2 \mathrm{~mm}, 1 \mathrm{~mm}, 0.5 \mathrm{~mm}$ and $0.25 \mathrm{~mm}$, and the mesh size of the 248 remaining surfaces is chosen as $3.2 \mathrm{~mm}$. The calculated SCFs of Specimen A are depicted in 249 Fig. 4. As can be seen, the SCFs converge at a mesh size of $0.5 \mathrm{~mm}$. Further refining mesh 250 size to $0.25 \mathrm{~mm}$ does not result to significant difference in the calculated SCFs. Therefore, the 251 mesh size of $0.5 \mathrm{~mm}$ is deemed as the appropriate size at the welding regions for calculating 252 SCFs. The created mesh is depicted in Fig. 5.
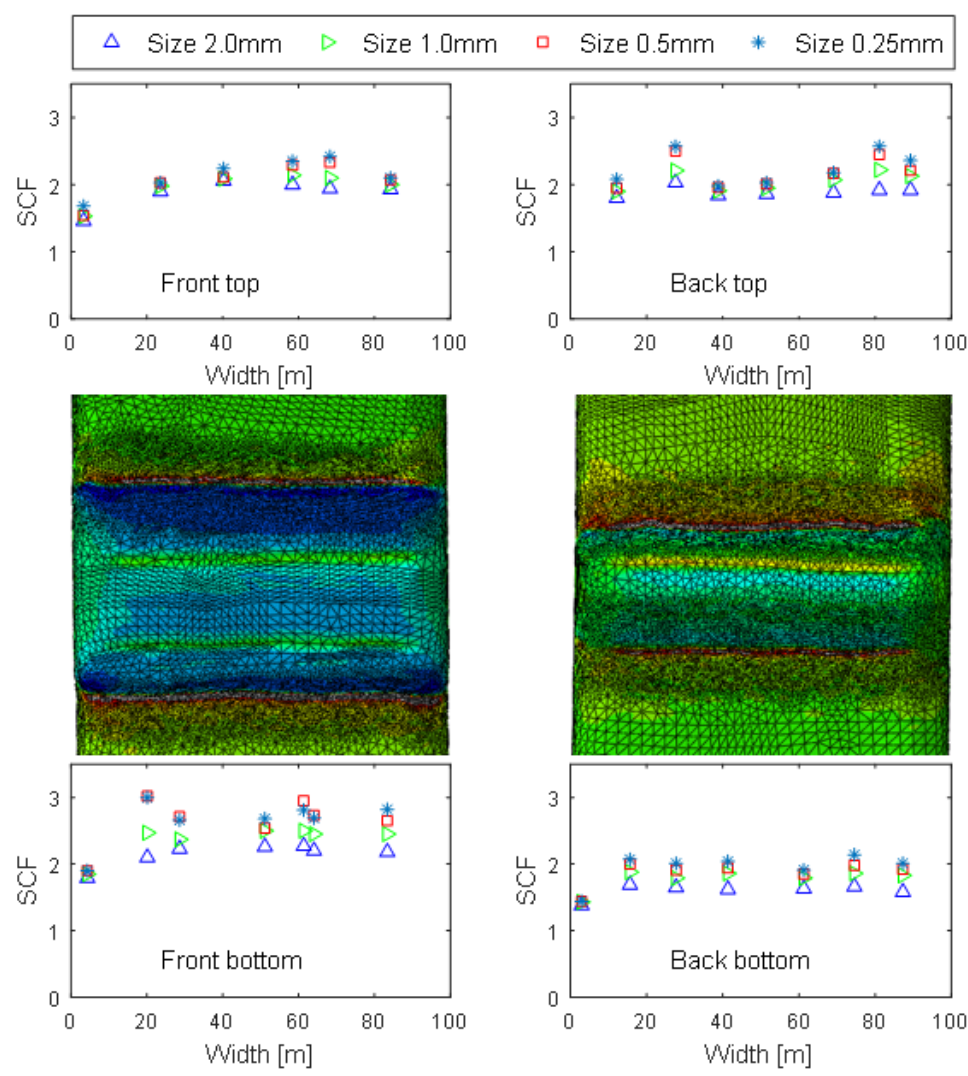

Figure 4. Mesh convergence (illustrated by results of Specimen A) 


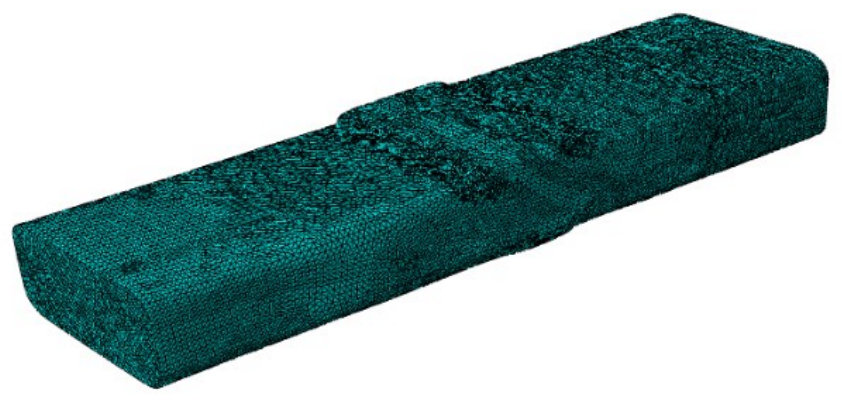

Figure 5. FEA Mesh

257

258

259

260

261

262

263

\subsubsection{Boundary conditions}

A z symmetric boundary condition, which is with respect to the created local coordinate system (see Fig. 3b in Section 2.2.1), is applied to one end of the specimen. During the experimental test in this work, specimens were tested under cyclic loading conditions with various load ranges. However, it should be noted that SCF is mainly dependent on the geometry of the welded joint, as it is the ratio of the local stress at the welded region to the nominal stress; hence, instead of applying cyclic loads, a uniformly distributed force with a total value of 100 $\mathrm{kN}$ is applied to the other end of the specimen. Fig. 6 presents the boundary conditions used in this study.

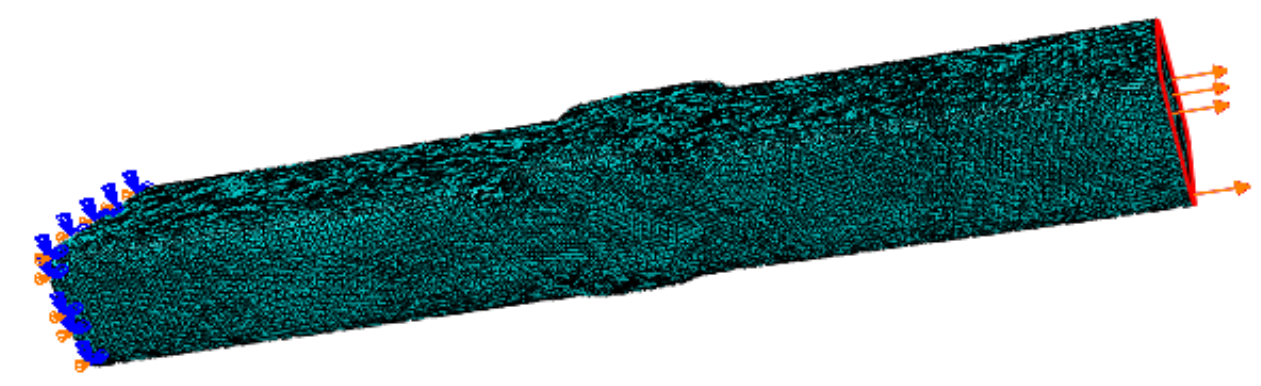

Figure 6. Boundary conditions

\subsubsection{FEA simulation and post-processing to derive SCFs}

Having defined geometry, material, mesh and boundary conditions, 3D FEA simulations were performed to calculate stresses in the welded joint. In this study, a static analysis is performed. After completing the simulations, the SCFs are then obtained through dividing local stresses by nominal stress. 
274 Fig. 7 depicts the flowchart of the LST-FEA3D model for calculating SCFs of welded specimens, which combines the 3D LST (presented in Section 2.1) and the 3D FEA (presented in Section 2.2).

279

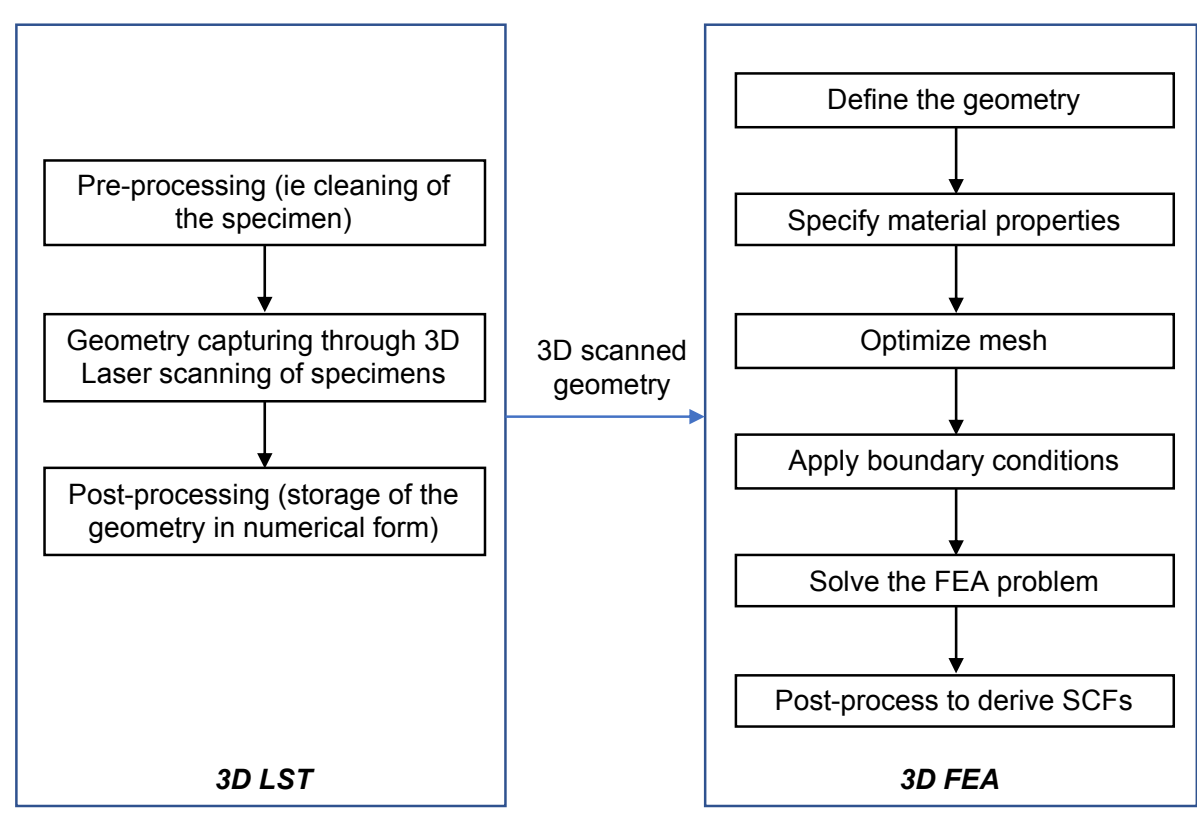

Figure 7. Flowchart of 3D LST-FEM model

\section{RSM-FEA2D (Combined 2D FEA and SRM)}

This section presents the development of the RSM-FEA2D model for the calculation of SCFs in offshore welded structures, which is derived by combining 2D FEA and RSM.

\subsection{D FEA modelling}

A parametric 2D FEA model for welded specimens is developed using ANSYS, which has been widely used in parametric FEA modelling. The geometry, material properties, mesh and boundary conditions used in the 2D FEA model are presented below.

\subsubsection{Geometry}

The geometry used in the 2D FEA model is presented in Fig. 8, from which it can be seen that the model includes two plates connected through the welding joint. 


\subsubsection{Material properties}

293

The material properties used in the 2D FEA modelling are identical to those used in 3D FEA modelling (see Section 2.2.2), i.e. S355 steel having Young's modulus of 210GPa and Poisson's ratio of 0.3 .

296

\subsubsection{Mesh}

297 In this study, ANSYS adaptive mesh function is used, in which the mesh is refined 298 automatically by ANSYS until the stress results satisfy the given convergence criterion. In this study, the stress variation of $1 \%$ is taken as the convergence criterion. An example of mesh convergence is presented in Table 1. As can be seen from Fig. 9, the stress reaches convergence at the $2^{\text {nd }}$ refinement, with a relative difference of $0.09 \%$ when compared to the previous solution.

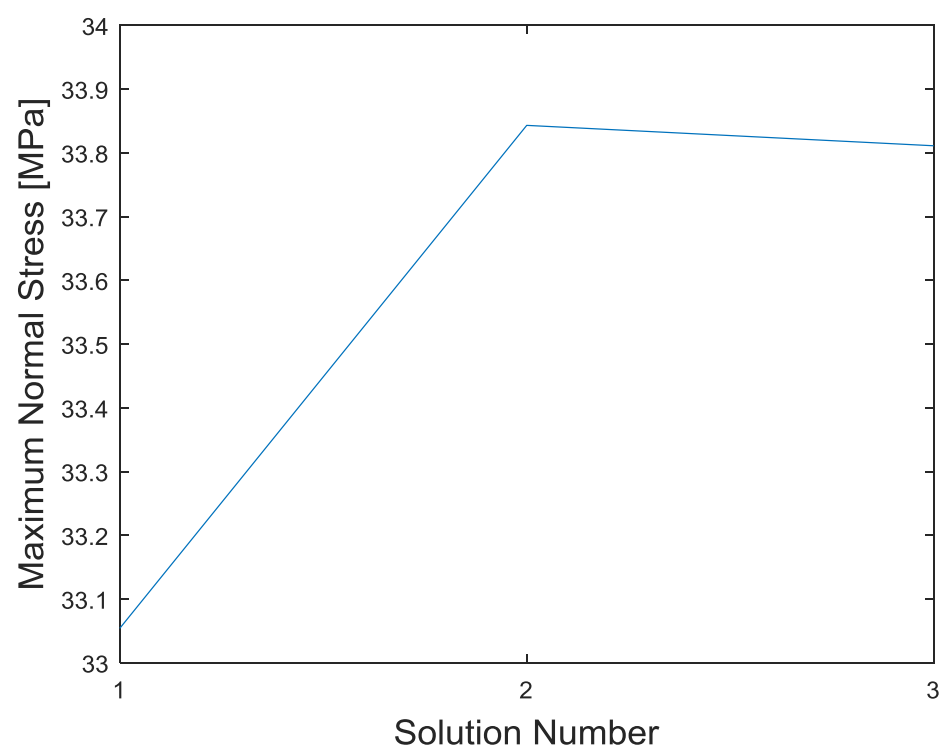




\begin{tabular}{ccccc}
\hline $\begin{array}{c}\text { Solution } \\
\text { number }\end{array}$ & $\begin{array}{c}\text { Maximum } \\
\text { normal stress } \\
\text { [MPa] }\end{array}$ & $\begin{array}{c}\text { Number of } \\
\text { elements }\end{array}$ & $\begin{array}{c}\text { Relative change } \\
{[\%]}\end{array}$ & $\begin{array}{c}\text { Mesh } \\
\text { description }\end{array}$ \\
\hline 1 & 33.05 & 4,872 & - & Initial mesh \\
2 & 33.84 & 43,234 & 2.39 & Refinement 1 \\
3 & 33.81 & 65,384 & 0.09 & Refinement 2 \\
\hline
\end{tabular}

\subsubsection{Boundary conditions}

A fixed support is applied to one end of the specimen, and a uniformly distributed force is applied to the other end. Fig. 10 depicts the boundary condition used in the 2D FEA modelling.

\section{A: Fixed support \\ B: Force}
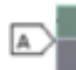

\subsection{Response surface method (RSM)}

RSM is a comprehensive statistical approach and has been widely used to approximate the relationship between independent and dependent variable(s) (Kolios et al., 2018). It can also be considered as the function interpolating or fitting discrete data points, which determines the deterministic formulas to describe the relationship of input and output of the concerned systems based on the observed samples in the design space. The discrete data points used in the RSM can be obtained from either experiments or numerical simulations. Once the response surface (i.e. the relationship between the input and output of the concerned systems) is established, the output of the concerned system with varied input can be efficiently obtained using the established relationship. RSM therefore saves much cost and time in experiments or numerical simulations by reducing the overall number of experiments or numerical simulations required.

In this study, the discrete data points used in the RSM are obtained by performing a finite number of deterministic 2D FEA simulations using the parametric 2D FEA model presented in 
using multivariate regression. In the general case of second-order polynomial regression 331 without mixed terms, the problem can be described as:

$$
y(x)=a_{0}+a_{1} x_{1}+a_{2} x_{1}^{2}+a_{3} x_{2}+a_{4} x_{2}^{2}+\cdots+a_{2 n-1} x_{n}+a_{2 n} x_{n}^{2}+e
$$

332

where $a_{0}$ and $a_{i}$ are the constant coefficient and constant exponents to be fitted.

where $Y$ is a matrix containing dependent variables; $X$ is a matrix containing independent variables; $A$ and $E$ are matrices with regression coefficients and error terms, respectively.

The regression coefficients $A$ in Eq. (2) can be obtained using the LSM (Least-Square Method):

$$
A=\left(X^{T} X\right)^{-1} X Y
$$

In addition to polynomial equations, other types of equations, can also be used in RSM. A such example is presented as follows.

$$
Y=1+a_{0} \prod_{i=1}^{n} X_{i}^{a_{i}}
$$

343

\subsection{Flowchart of RSM-FEA2D model}

Fig. 11 presents the flowchart of the RSM-FEA2D model for calculating SCFs, which combines the 2D FEA (presented in Section 3.1) and the RSM (presented in Section 3.2). 


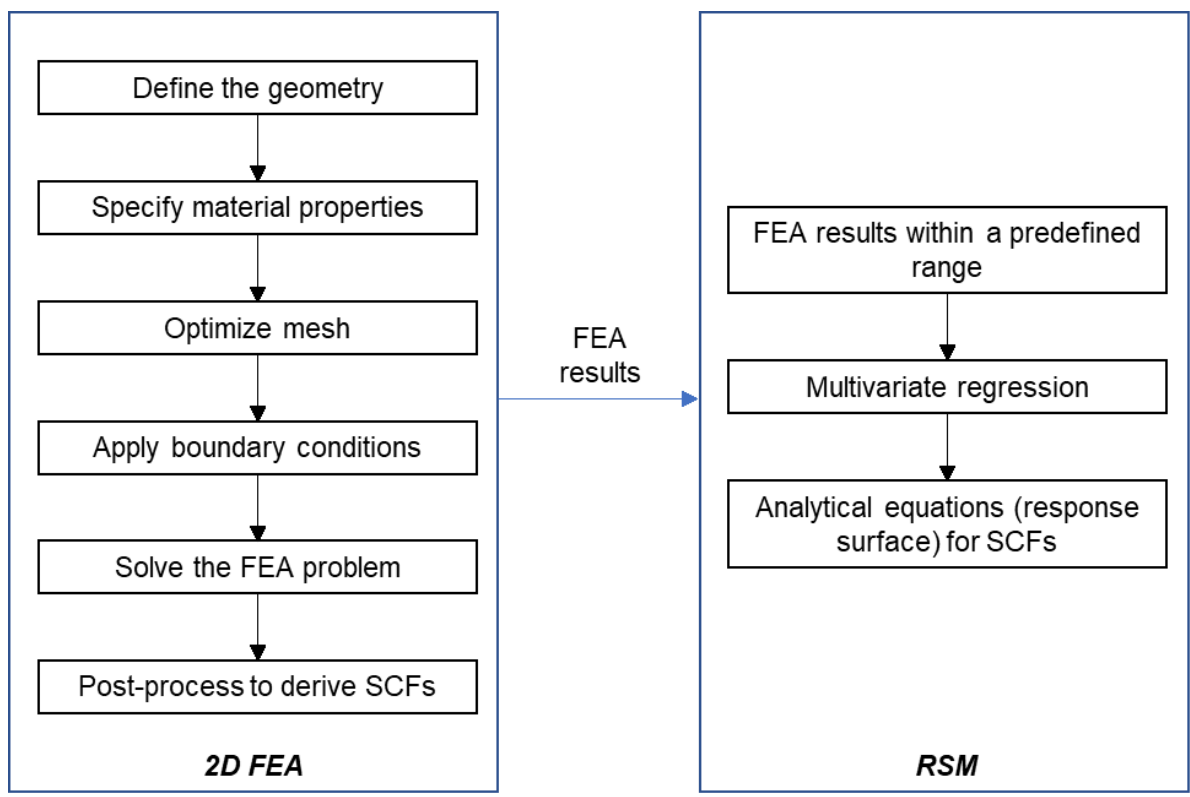

Figure 11. Flowchart of RSM-FEA2D model for calculating SCFs

\section{Results and discussion}

350 The LST-FEA3D and RSM-FEA2D models for calculating SCFs of offshore wind monopile 351 structures welded specimens are developed based on the flowchart presented in Figs. 7 and 35211 , respectively. A number of case studies are performed to validate the RSM-FEA2D model. 353 After the validation, the RSM-FEA2D model is then applied to the geometries of the tested specimens to derive analytical equations for calculating SCFs. The LST-FEA3D model is also applied to the tested large scale specimens, obtaining detailed SCF distributions along the welding regions.

\subsection{Testing of large scale steel specimens}

The specimens that were tested in this programme were of a dog-bone geometry, made of S355 steel. The specimens were fabricated initially through welding of two plates (blanks) of $50 \mathrm{~mm}$ thickness with double-butt weld and then were machined into the dog-bone geometries. Sharp edges were ground and shot pinned in order to avoid invalid test specimens with cracks in places other than the weld region. The specimens were tested axially (tensiontension) under cyclic loading conditions. Fig. 12 illustrates the experimental configuration with purpose-designed mechanical grips. Before commencement of any testing, the test machine Load Cell was calibrated dynamically according to relevant design standards to a level 1.5 times the maximum test load. 


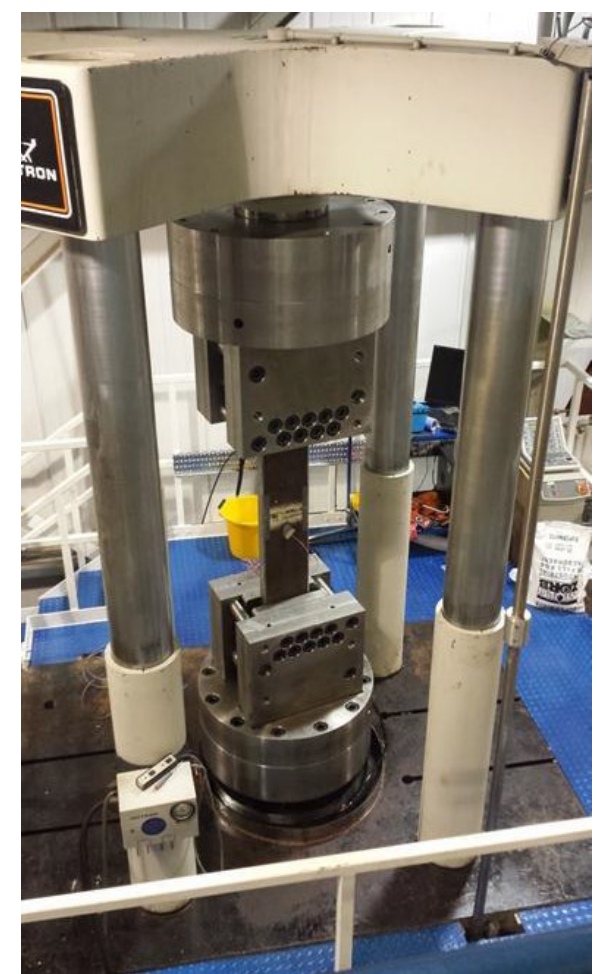

Figure 12. Experimental configuration of large scale dog-bone tests

\subsection{Validation of RSM-FEA2D}

370

A case study is performed to validate the RSM-FEA2D model. In this case, a plate with a hole geometry (see Fig. 13) is taken as a reference geometry. The centre of the hole is located at the centre of the plate, and the geometry parameters are listed in Table 2.

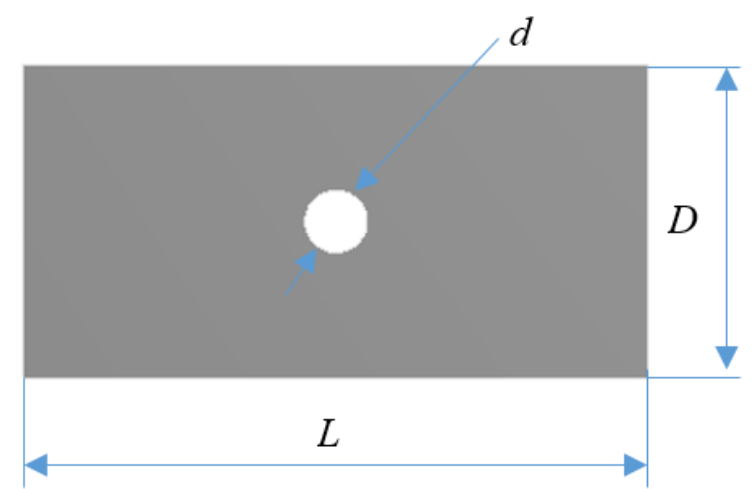

Figure 13. Geometry of plate with a hole 


\begin{tabular}{lll}
\hline Item & Value & Description \\
\hline$D[\mathrm{~m}]$ & 5 & Height \\
$L[\mathrm{~m}]$ & 10 & Length \\
$d[\mathrm{~m}]$ & {$[0.1 D 0.9 D]$} & Hole diameter \\
\hline
\end{tabular}

378

379 In this case, the left end of the plate is fixed, and the right end of the plate is loaded with a 380 force of $1 \mathrm{MN}$, as illustrated in Fig. 14.

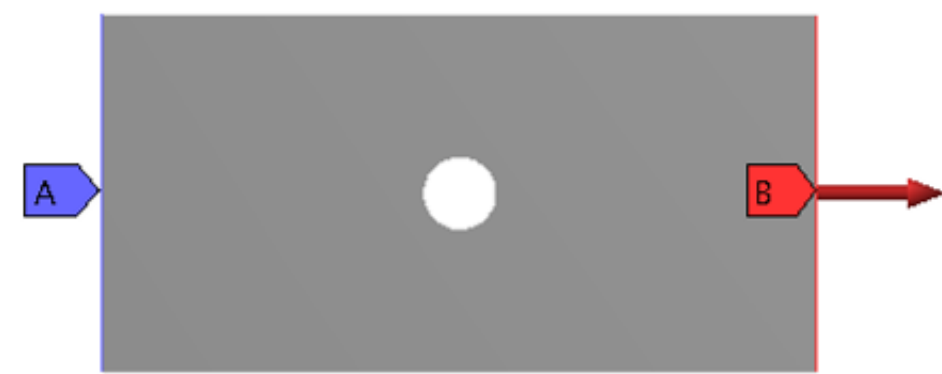

382

Figure 14. Boundary conditions

The diameter of the hole, $d$, is considered as a variable. 1,000 random samples are generated by randomly changing the values of $d$ within given limits of $[0.1 D-0.9 D]$, i.e. [0.5 $m-0.45 m$ ] in case $D=5 \mathrm{~m}$. With 1,000 samples, 1,000 FEA simulations have been performed, obtaining 1,000 data set as illustrated in Table 3.

\begin{tabular}{|c|c|c|c|c|c|}
\hline Sample ID & $d[\mathrm{~m}]$ & $\sigma_{\max }[\mathrm{Pa}]$ & $\sigma_{\text {nominal }}[\mathrm{Pa}]$ & $1-d / D[\mathrm{~m}]$ & SCF \\
\hline 1 & 2.500 & $8,749,054$ & $4,000,000$ & 0.500 & 2.190 \\
\hline 2 & 4.500 & $40,971,542$ & $19,987,595$ & 0.100 & 2.050 \\
\hline 3 & 0.500 & $6,128,614$ & $2,222,414$ & 0.900 & 2.760 \\
\hline 4 & 1.500 & $6,779,455$ & $2,857,232$ & 0.700 & 2.370 \\
\hline 5 & 3.500 & $14,047,815$ & $6,667,054$ & 0.300 & 2.110 \\
\hline$\ldots$ & $\ldots$ & $\ldots$ & $\ldots$ & $\ldots$ & . \\
\hline 1000 & 2.380 & $8,403,617$ & $3,813,760$ & 0.520 & 2.200 \\
\hline
\end{tabular}
following equation for SCF: 


$$
S C F=1.993+0.6387 x-1.431 x^{2}+1.86 x^{3}
$$

where

$$
x=(1-d / D)
$$

392 393

394

395

396

397

$$
S C F=2+0.284(1-d / D)-0.6(1-d / D)^{2}+1.32(1-d / D)^{3}
$$

398 The SCF calculated from Eq. (5), which is derived by RSM-FEA2D, is compared against the 399 SCF calculated from Eq. (7) given in Ref. (Walter D. Pilkey, 2008), as illustrated in Fig. 16. In 400 this case, the plate width $D$ is fixed with a value of $5 \mathrm{~m}$, and the hole diameter $d$ varies between $4010.5 \mathrm{~m}$ and $0.45 \mathrm{~m}$. As can be seen from Fig. 16, the SCF obtained from the combined 2D FEA 402 403 404 in Fig. 15.

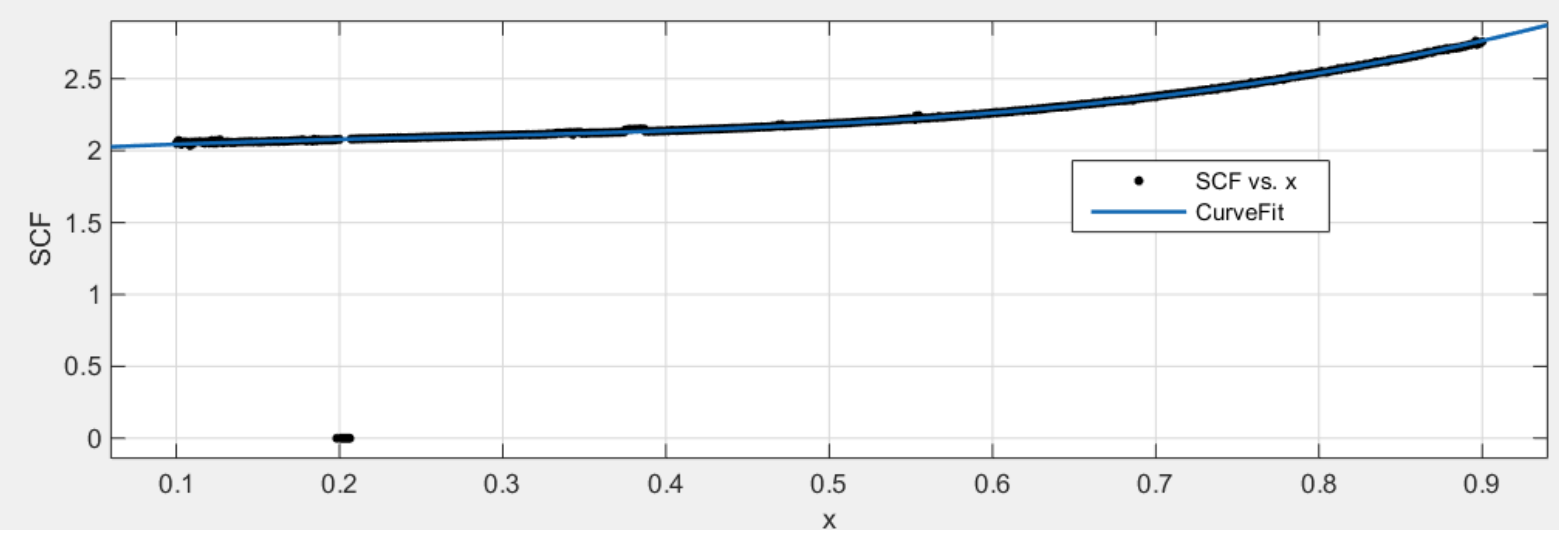

Figure 15. Curve fit

The following expression of SCF for plate with a hole is taken from Ref. (Walter D. Pilkey, 2008): and RSM show good agreement with the SCF obtained from Ref. (Walter D. Pilkey, 2008), with a maximum relative difference $(1.9 \%)$ observed at hole of diameter of $3.5 \mathrm{~m}$. This confirms the validity of the RSM-FEA2D model developed in this work. 
405

406

407

408

409

410
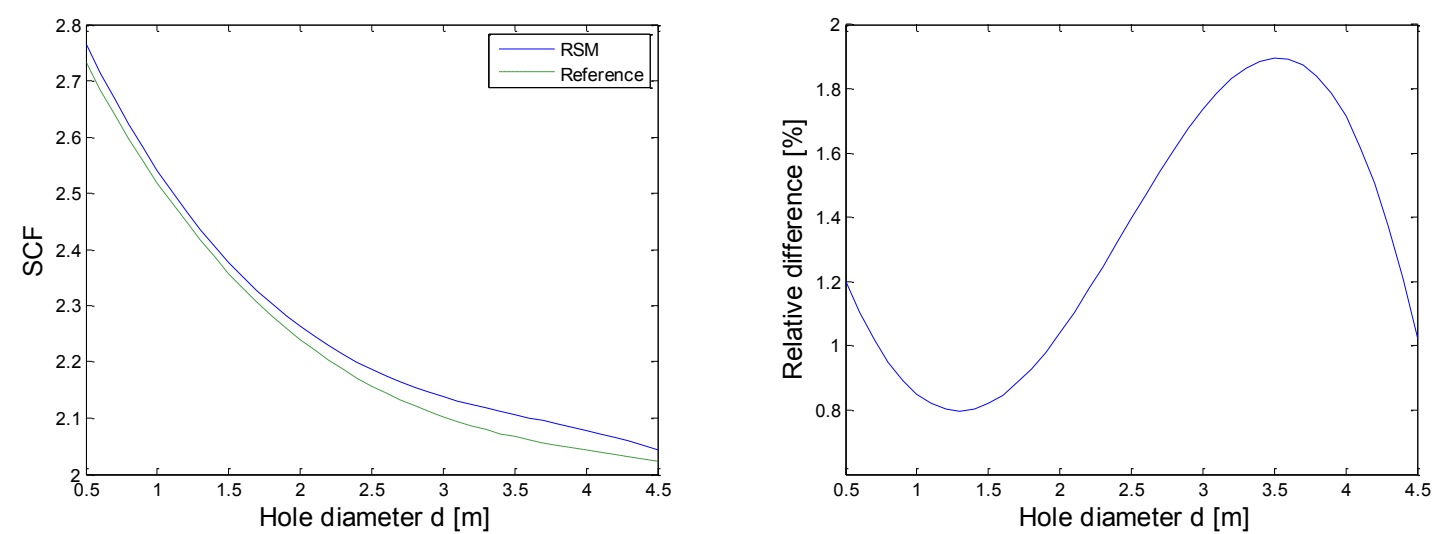

Figure 16. Calculated SCF: a Comparison of SCF obtained from RSM and Reference; $\mathbf{b}$

Relative difference

\subsection{SCF equations derived from RSM-FEA2D}

The RSM-FEA2D model is applied to the geometries of tested specimens to derive the analytical equation applicable for SCFs for offshore wind monopile welded structures. The geometry of the $2 \mathrm{D}$ cross-section of the welded specimens used in the $2 \mathrm{D}$ FEA model is presented in Fig. 17. As can be seen, the geometry is defined through five parameters, i.e. thickness $t$, length $L$, weld height $h$, weld angle $\theta$ and weld toe radius $r$. The values of these five parameters are listed in Table 4.

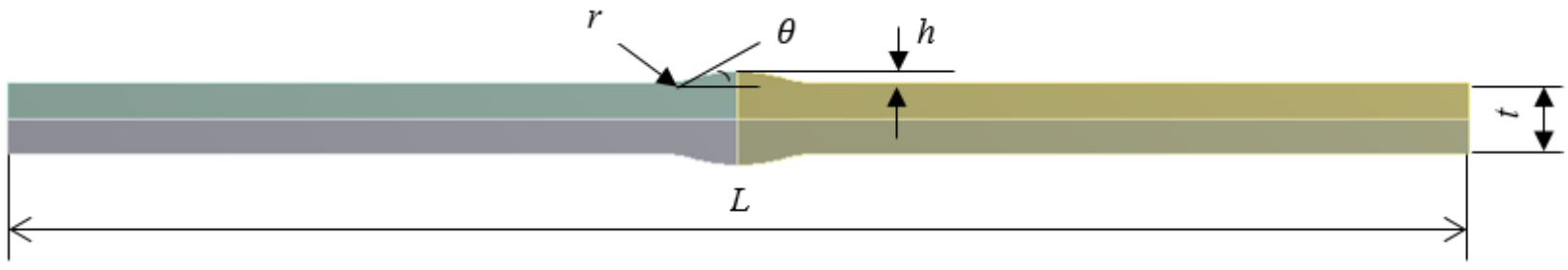

Figure 17. Geometric parameters of 2D cross-section of typical welded specimens

Table 4. Geometry parameters

\begin{tabular}{lll}
\hline Item & Value & Description \\
\hline$t[\mathrm{~m}]$ & {$[0.010 .1]$} & Plate thickness \\
$L[\mathrm{~m}]$ & 1 & Plate length \\
$h / t[-]$ & {$[0.010 .1]$} & $\begin{array}{l}\text { Ratio of weld height } h \text { to } \\
\text { plate thickness } t\end{array}$ \\
$\theta$ [deg.] & & Weld angle \\
$r[\mathrm{~m}]$ & {$[1045]$} & Weld toe radius \\
\hline
\end{tabular}


419 All parameters in Table 4 except for plate length affect the value of SCF. Therefore, the plate 420 length in this case is treated as constant, while the plate thickness, ratio of weld height to plate 421 thickness $h / t$, weld angle $\theta$ and weld toe radius $r$ are considered as variables. 1,000 random 422 samples are generated by randomly changing the values of these three variables within the 423 given limit specified in Table 4.

424 The 1,000 FEA simulation results are then post-processed through regression. Two forms of 425 equations are investigated, i.e. second-order polynomial equation and multivariate power-law 426 equation. The SCF expression derived in the form of second-order polynomial is expressed 427 as:

$$
\begin{aligned}
S C F=1.299+ & 7.486\left(\frac{h}{t}\right)-1.117\left(\frac{r}{t}\right)+0.406 \tan \theta-34.279\left(\frac{h}{t}\right)^{2}+0.440\left(\frac{r}{t}\right)^{2} \\
& -0.325(\tan \theta)^{2}
\end{aligned}
$$

The SCF expression derived in the form of multivariate power-law equation is expressed as:

$$
S C F=1+0.720\left(\frac{h}{t}\right)^{0.438}\left(\frac{r}{t}\right)^{-0.458}(\tan \theta)^{0.118}
$$

429 The derived SCF expression in Eq. (8) with the second-order polynomial form has a $R^{2}$ value 430 of 0.656 , which is relatively low and indicates Eq. (8) does not fit the original data well. The 431 derived SCF expression in Eq. (9) with the multivariate power-law form has a $R^{2}$ value of 4320.970 , which indicates a high accuracy regression. This indicates that the form of expression used in the regression process can significantly affect the accuracy of regression. Eq. (9) has high accuracy and it is therefore chosen as the analytical expression for SCF in this work.

Fig. 18 presents the comparison of the predicted data, which are obtained using Eq. (9), and the original data, which are obtained from the 1,000 FEA simulations. As can be observed, the predicted data show reasonable agreement with the original data. Eq. (9) derived in this work can be therefore used confidently to effectively calculate the SCF of welded specimens. 


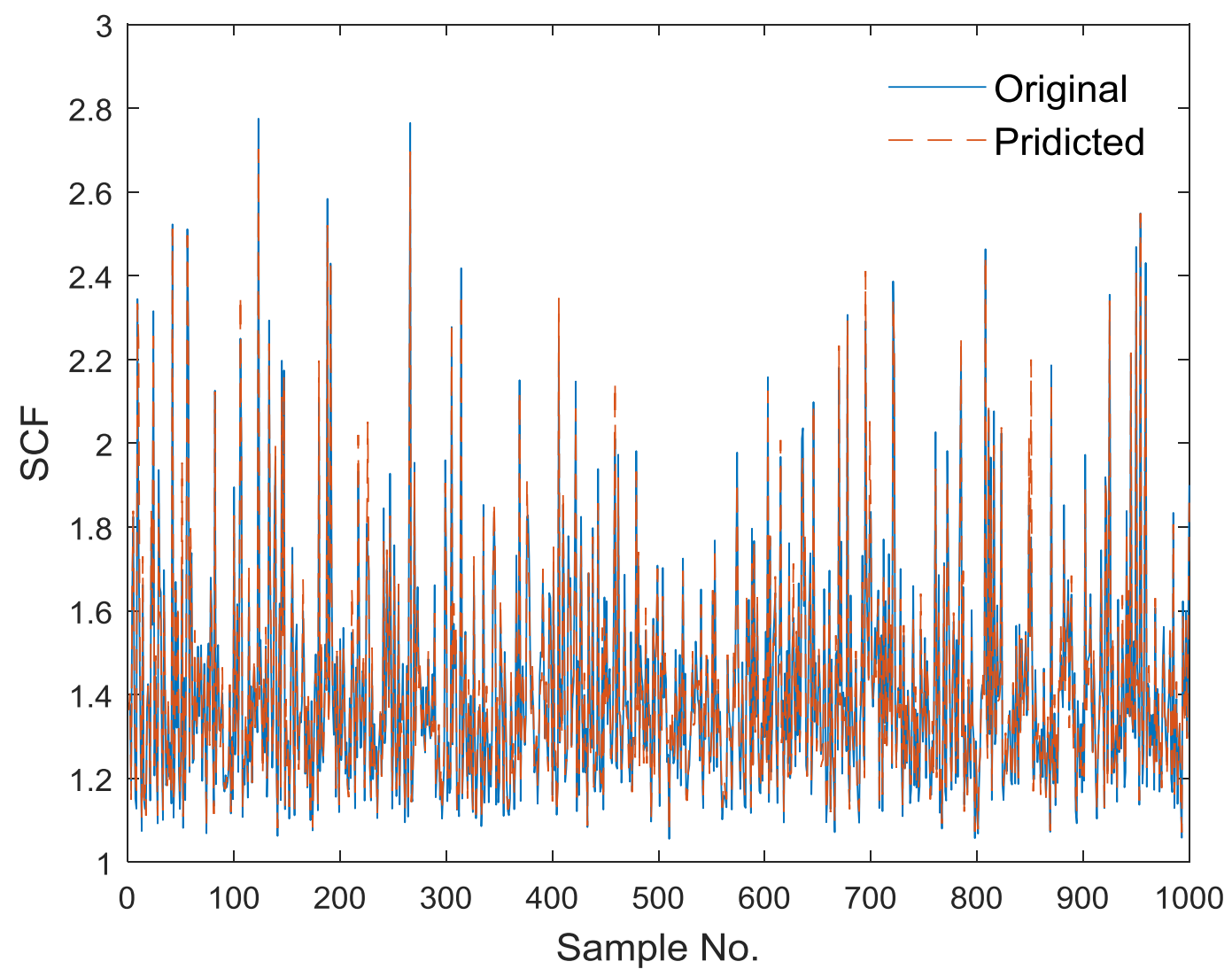

441

Figure 18. Comparison of original and predicted data

442 In Ref. (He and Zhang, 2011), a SCF expression for double-V butt welded joint was proposed 443 for a specific case with weld toe angle of $30^{\circ}$, plate thickness of $0.01 \mathrm{~m}$, and it is expressed as:

$$
S C F=0.817 e^{-r / 1.013}+0.482 e^{-r / 9.717}+0.629 e^{-r / 0.215}+1.038
$$

444 where $r$ in Eq. (10) is the weld toe radius. It should be noted that $r$ in Eq. (10) is in mm, while 445 the $r$ in Eq. (9) is in $\mathrm{m}$.

446 The SCFs calculated using Eq. (9) derived in this work are compared against the SCFs 447 calculated using Eq. (10), and the comparison results are depicted in Fig. 19. In this case, 448 weld toe angle is $30^{\circ}$, plate thickness is $0.01 \mathrm{~m}$ and ratio of weld height to plate thickness is 4490.08 , and the weld toe radius varies between $0.001 \mathrm{~m}$ and $0.008 \mathrm{~m}$. 


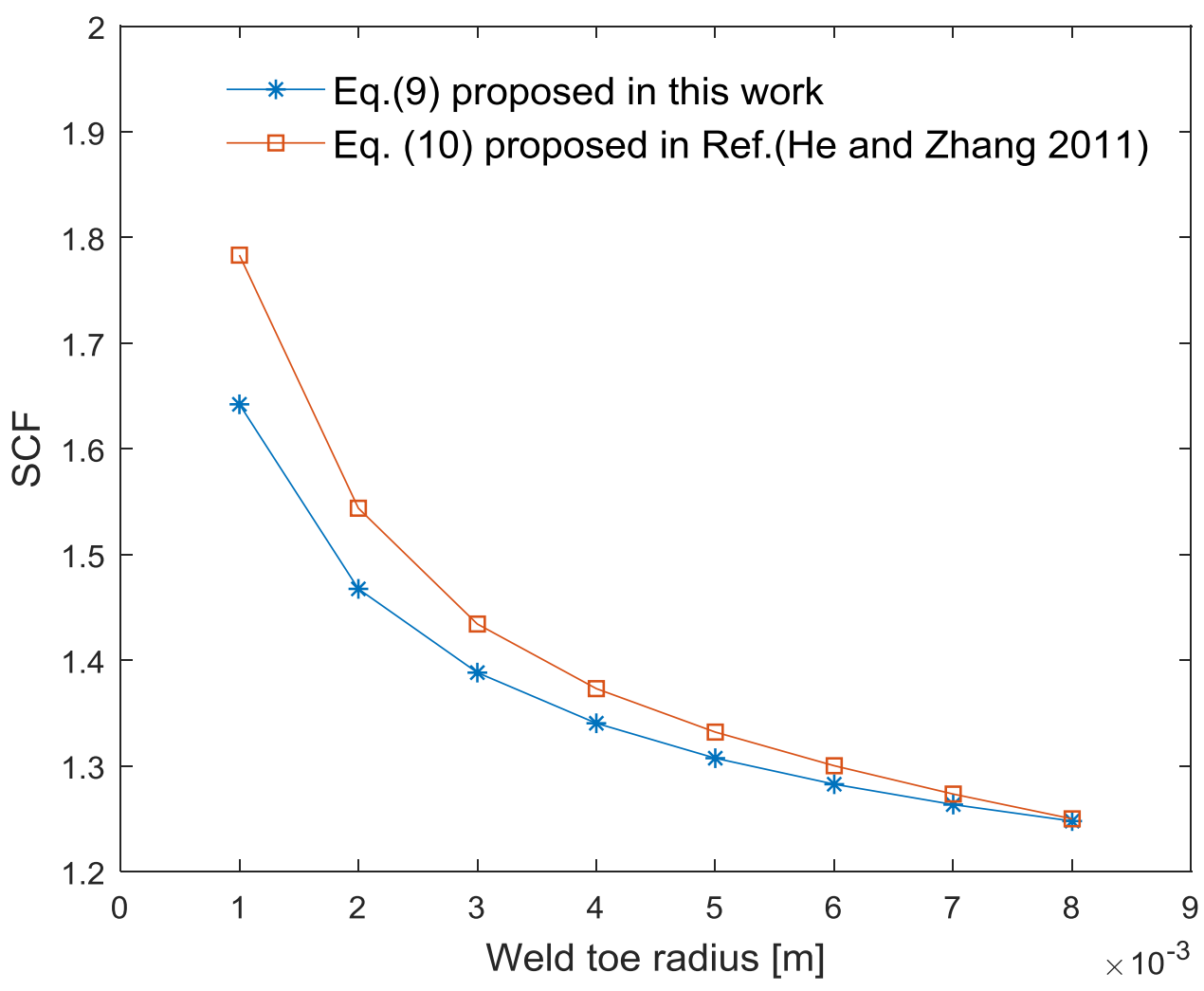

452 As can be seen from Fig. 19, the results from Eq. (9) derived in this work show reasonable 453 agreement with those from Eq. (10) proposed in Ref. (He and Zhang, 2011), with a maximum relative difference $(7.9 \%)$ observed at weld toe radius of $0.001 \mathrm{~m}$. This further confirms the validity of the SCF equation derived in this work. Compared to Eq. (10) proposed in Ref. (He and Zhang, 2011), which is limited to a single weld toe angle and plate thickness, Eq. (9) derived in this work is more comprehensive as it takes account of all parameters associated with SCF for double-V butt weld joints.

\subsection{SCF distributions calculated with LST-FEA3D}

The LST-FEA3D model is also applied to the geometries of the tested specimens to calculate SCFs, investigating the distribution of SCFs along the width of the welded specimens. In this work, more than 30 specimens were experimentally tested and LST-FEA3D model has been applied to all tested specimens to calculate SCFs. The variations in manufactured specimens 
466 makes it inevitable to have some variations in actual welding profile of manufactured 467 specimens, although all specimens were manufactured based on the same technical 468 specification. The manufactured specimens were experimentally tested under various load 469 ranges. In this case, Specimens $B$ and C, which were tested under load range of $1295 \mathrm{kN}$ and $4701100 \mathrm{kN}$ respectively, are chosen as two examples. Figs. 20 and 21 present the distributions 471 of SCFs along the welding region of Specimens B and C, respectively. From these figures it 472 can be seen that 1) stress concentration is observed along the welding regions, as expected; 473 2) SCFs fluctuate significantly along the welding regions; 3 ) the critical point, where fatigue 474 cracking is most likely to initiate, in Specimen B is observed at its front bottom corresponding 475 to the peak SCF of $2.50 ; 4)$ the critical point in Specimen C is observed at front top 476 corresponding to the peak SCF of 2.27.
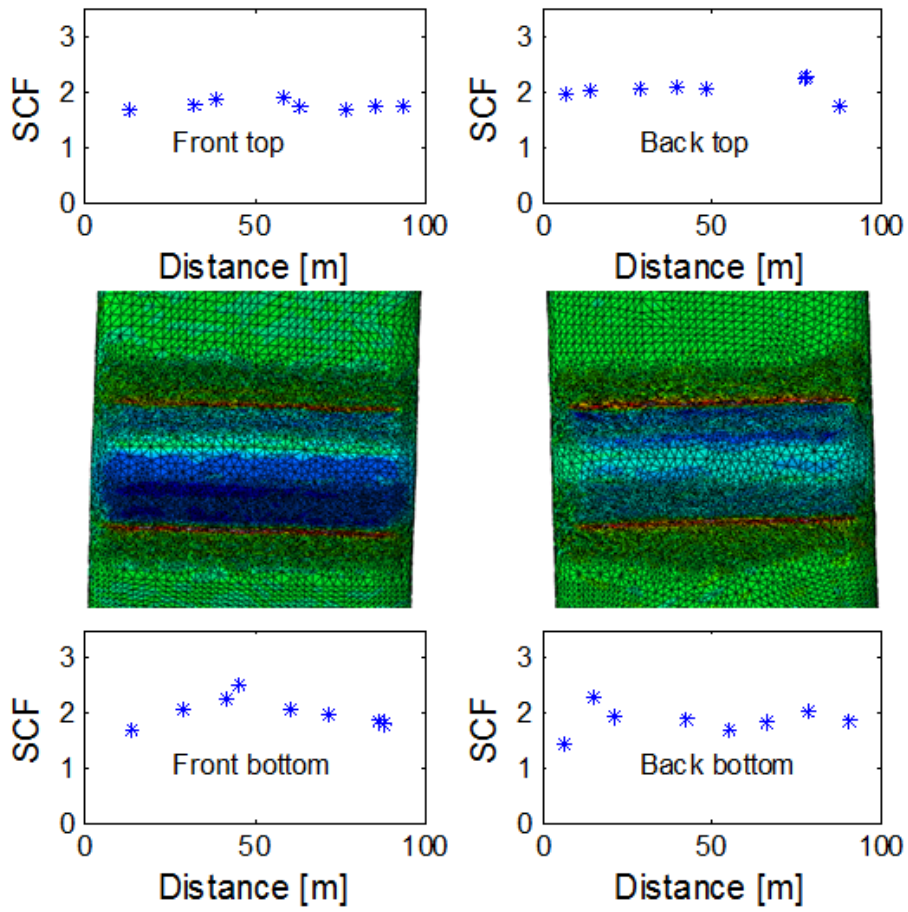

Figure 20. Distributions of SCFs of Specimen B 

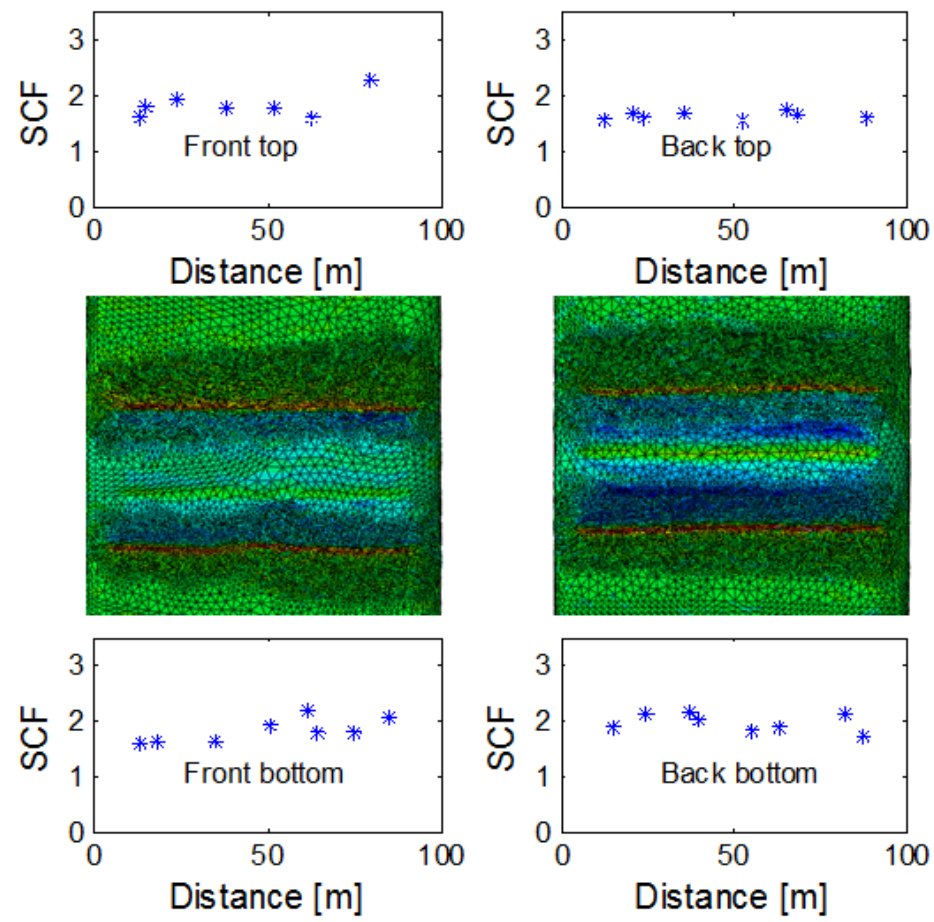

Figure 21. Distributions of SCFs of Specimen C

\section{Conclusions}

482

In this study a hybrid experimental and numerical model has been successfully developed for calculating SCFs in offshore welded structures, i.e. LST-FEA3D model and RSM-FEA2D model. The LST-FEA3D model is developed by combining the 3D (three-dimensional) LST (laser scan technology) and 3D FEA (finite element analysis). In this model, the 3D LST is used to obtain the precise geometry of the welded specimens, retaining high detail of welding profiles. The RSM-FEA2D model is developed by combining the non-linear RSM (response surface method) and the 2D FEA. In this model, a parametric 2D FEA model of butt-welded specimens is developed. A number of FEA simulations are performed by randomly varying the weld parameters within given limits. Multivariate regression is then used to post-process the FEA results, establishing the response surface. Both LST-FEA3D and RSM-FEA2D models are applied to typical offshore wind monopile welded steel specimens tested to failure under cyclic loading. The following conclusions can be derived from the present study:

1) The analytical equation derived from the present RSM-FEA2D model, which has been validated through case studies, can efficiently calculate the SCFs of offshore welded monopile specimens. 
2) The results from the LST-FEA3D model indicate that the SCFs along the welding region are not constant and show nonlinear distribution. This behaviour cannot be captured by conventional methods for calculating SCFs, such as empirical equations suggested by the design standards and the RSM-FEA2D model.

3) The LST-FEA3D model is demonstrated to be capable of obtaining high detail of the welding profiles and calculating SCFs along the welding regions as well as identifying the critical point where fatigue cracking is most likely to initiate.

\section{ACKNOWLEDGEMENT}

The authors would like to acknowledge the input of Mr Antony Charnley and Dr Wilson Vesga who carried out the experimental elements of this work.

\section{References}

Ahmadi, H., 2016. A probability distribution model for SCFs in internally ring-stiffened tubular KT-joints of offshore structures subjected to out-of-plane bending loads. Ocean Eng. 116, 184-199. https://doi.org/10.1016/j.oceaneng.2016.02.037

Ahmadi, H., Lotfollahi-Yaghin, M.A., 2015. Stress concentration due to in-plane bending (IPB) loads in ring-stiffened tubular KT-joints of offshore structures: Parametric study and design formulation. Appl. Ocean Res. 51, 54-66. https://doi.org/10.1016/j.apor.2015.02.009

Ahmadi, H., Lotfollahi-Yaghin, M.A., 2013. Effect of SCFs on S-N based fatigue reliability of multi-planar tubular DKT-joints of offshore jacket-type structures. Ships Offshore Struct. 8, 55-72. https://doi.org/10.1080/17445302.2011.627750

Ahmadi, H., Lotfollahi-Yaghin, M.A., Aminfar, M.H., 2011a. Geometrical effect on SCF distribution in uni-planar tubular DKT-joints under axial loads. J. Constr. Steel Res. 67, 1282-1291. https://doi.org/10.1016/j.jcsr.2011.03.011

Ahmadi, H., Lotfollahi-Yaghin, M.A., Aminfar, M.H., 2011b. Effect of stress concentration factors on the structural integrity assessment of multi-planar offshore tubular DKT-joints based on the fracture mechanics fatigue reliability approach. Ocean Eng. 38, 1883-1893. https://doi.org/10.1016/j.oceaneng.2011.08.004

Ahmadi, H., Lotfollahi-Yaghin, M.A., Yong-Bo, S., 2013. Chord-side SCF distribution of central brace in internally ring-stiffened tubular KT-joints: A geometrically parametric study. Thin- 
Ahmadi, H., Yeganeh, A., Mohammadi, A.H., Zavvar, E., 2016. Probabilistic analysis of stress concentration factors in tubular KT-joints reinforced with internal ring stiffeners under inplane bending loads. Thin-Walled Struct. 99, 58-75. https://doi.org/10.1016/j.tws.2015.11.010

Ahmadi, H., Zavvar, E., 2016. The effect of multi-planarity on the SCFs in offshore tubular KTjoints subjected to in-plane and out-of-plane bending loads. Thin-Walled Struct. 106, 148-165. https://doi.org/10.1016/j.tws.2016.04.020

American Petroleum Institute, 2014. Recommended Practice 2A-WSD for Planning, Designing, and Constructing Fixed Offshore Platforms - Working Stress Design.

Cao, Y., Meng, Z., Zhang, S., Tian, H., 2013. FEM study on the stress concentration factors of K-joints with welding residual stress. Appl. Ocean Res. 43, 195-205. https://doi.org/10.1016/j.apor.2013.09.006

Christiansen, N.H., Tang, B.K., 2016. Neural Networks for Tubular Joint Optimization in Offshore Jacket Structures, in: Volume 1: Offshore Technology; Offshore Geotechnics. ASME, p. V001T01A013. https://doi.org/10.1115/OMAE2016-54846

Chung, J., Wallerand, R., Hélias-Brault, M., 2013. Pile Fatigue Assessment During Driving. Procedia Eng. 66, 451-463. https://doi.org/10.1016/j.proeng.2013.12.098

DNV GL AS, 2016. DNVGL-ST-0126 Support structures for wind turbines.

E. Angelopoulou and J. R. Wright Jr, 1999. Laser scanner technology. Philadelphia, PA, USA.

EWEA, 2015. Wind energy scenarios for 2030. Brussels, Belgium.

Gentils, T., Wang, L., Kolios, A., 2017. Integrated structural optimisation of offshore wind turbine support structures based on finite element analysis and genetic algorithm. Appl. Energy 199. https://doi.org/10.1016/j.apenergy.2017.05.009

He, B.L., Zhang, X.D., 2011. Finite Element Calculation about Stress Concentration Coefficient of Welded Butt Joints Based on the ABAQUS. Appl. Mech. Mater. 80-81, 807-811. https://doi.org/10.4028/www.scientific.net/AMM.80-81.807

Igwemezie, V., Mehmanparast, A., Kolios, A., 2018. Materials selection for XL wind turbine 
support structures: A corrosion-fatigue perspective. Mar. Struct. 61, 381-397. https://doi.org/10.1016/j.marstruc.2018.06.008

loannou, A., Angus, A., Brennan, F., 2018. A lifecycle techno-economic model of offshore wind energy for different entry and exit instances. Appl. Energy 221, 406-424. https://doi.org/10.1016/j.apenergy.2018.03.143

J. Beraldin, F. Blais, and U.L., 2010. Laser scanning technology, in: Airborne and Terrestrial Laser Scanning.

Kallehave, D., Byrne, B.W., LeBlanc Thilsted, C., Mikkelsen, K.K., 2015. Optimization of monopiles for offshore wind turbines. Philos. Trans. R. Soc. A Math. Phys. Eng. Sci. 373, 20140100-20140100. https://doi.org/10.1098/rsta.2014.0100

Kolios, A., Collu, M., Chahardehi, A., Brennan, F.P., Patel, M.H., 2010. A multi-criteria decision making method to compare support structures for offshore wind turbines, in: European Wind Energy Conference and Exhibition 2010, EWEC 2010.

Kolios, A., Di Maio, L.F., Wang, L., Cui, L., Sheng, Q., 2018. Reliability assessment of pointabsorber wave energy converters. Ocean Eng. 163, 40-50. https://doi.org/10.1016/j.oceaneng.2018.05.048

Kolios, A., Mytilinou, V., Lozano-Minguez, E., Salonitis, K., 2016. A comparative study of multiple-criteria decision-making methods under stochastic inputs. Energies 9. https://doi.org/10.3390/en9070566

Lee, M., 1999. Estimation of stress concentrations in single-sided welds in offshore tubular joints. Int. J. Fatigue 21, 895-908. https://doi.org/10.1016/S0142-1123(99)00083-3

Li, Y., Zhou, X.-P., Qi, Z.-M., Zhang, Y.-B., 2014. Numerical study on girth weld of marine steel tubular piles. Appl. Ocean Res. 44, 112-118. https://doi.org/10.1016/j.apor.2013.11.005

Lozano-Minguez, E., Brennan, F.P., Kolios, A.J., 2014. Reanalysis of offshore T-joint fatigue life predictions based on a complete weld profile model. Renew. Energy 71. https://doi.org/10.1016/j.renene.2014.05.064

Lozano-Minguez, E., Kolios, A.J., Brennan, F.P., 2011. Multi-criteria assessment of offshore wind turbine support structures. Renew. Energy 36. https://doi.org/10.1016/j.renene.2011.04.020 
Maheswaran, J., Siriwardane, S.C., 2016. Fatigue life estimation of tubular joints - a comparative study. Fatigue Fract. Eng. Mater. Struct. 39, 30-46. https://doi.org/10.1111/ffe.12314

Mehmanparast, A., Taylor, J., Brennan, F., Tavares, I., 2018. Experimental investigation of mechanical and fracture properties of offshore wind monopile weldments: SLIC interlaboratory test results. Fatigue Fract. Eng. Mater. Struct. https://doi.org/10.1111/ffe.12850

Mehmanparast, A., O. Adedipe, F. Brennan, 2016. Welding sequence effects on residual stress distribution in offshore wind monopile structures. Frat. ed Integritá Strutt. https://doi.org/10.3221/IGF-ESIS.35.15

Méndez, G.T., Cuamatzi-Meléndez, R., Hernández, A.A., Capula-Colindres, S.I., AngelesHerrera, D., Velázquez, J.C., Vazquez-Hernández, O., 2017. Correlation of Stress Concentration Factors for T-Welded Connections - Finite Element Simulations and Fatigue Behavior. Soldag. Inspeção 22, 194-206. https://doi.org/10.1590/0104$9224 / \mathrm{si} 2202.08$

Ogeman, V., Mao, W., Ringsberg, J.W., 2014. Uncertainty in Stress Concentration Factor Computation for Ship Fatigue Design, in: Volume 4A: Structures, Safety and Reliability. ASME, p. V04AT02A008. https://doi.org/10.1115/OMAE2014-23129

Peeringa, K.H. and J., 2016. Future XL monopile foundation design for a $10 \mathrm{MW}$ wind turbine in deep water.

Pirali, M.H. and H., 2006. Hot Spot Stress Determination for a Tubular T-Joint under Combined Axial and Bending Loading. IUST Int. J. Eng. Sci. 17, 21-28.

Schmidt, D., Manuel, L., Nguyen, H.H., Sagrilo, L.V.S., Prates de Lima, E.C., 2015. Fatigue Reliability Analysis for Brace-Column Connection Details in a Semisubmersible Hull 1. J. Offshore Mech. Arct. Eng. 137, 061301. https://doi.org/10.1115/1.4031493

Seidel, M., 2010. Feasibility of monopiles for large offshore wind turbines, in: Proceedings of the 10th German Wind Energy Conference (DEWEK). REpower Systems AG. Germany.

Walter D. Pilkey, D.F.P., 2008. Peterson's Stress Concentration Factors, 3rd Edition.

Wu, J., Chen, N.-Z., 2017. Fracture Mechanics Based Fatigue Assessment for a Spar-Type 

V010T09A048. https://doi.org/10.1115/OMAE2017-61568

615

616

617

618

619

620

621

622

Yang, J., Chen, Y., Hu, K., 2015. Stress concentration factors of negative large eccentricity tubular $\mathrm{N}$-joints under axial compressive loading in vertical brace. Thin-Walled Struct. 96, 359-371. https://doi.org/10.1016/j.tws.2015.08.027

Zhang, J., Jiang, J., Shen, W., Luo, Y., 2018. A novel framework for deriving the unified SCF in multi-planar overlapped tubular joints. Mar. Struct. 60, 72-86. https://doi.org/10.1016/j.marstruc.2018.03.008 